\title{
Solar energy generation in three dimensions $\dagger$
}

\author{
Marco Bernardi, ${ }^{a}$ Nicola Ferralis, ${ }^{a}$ Jin H. Wan, ${ }^{b}$ Rachelle Villalon ${ }^{c}$ and Jeffrey C. Grossman ${ }^{* a}$
}

Received 20th January 2012, Accepted 21st February 2012

DOI: $10.1039 / \mathrm{c} 2 \mathrm{ee} 21170 \mathrm{j}$

We formulate, solve computationally and study experimentally the problem of collecting solar energy in three dimensions. We demonstrate that absorbers and reflectors can be combined in the absence of sun tracking to build three-dimensional photovoltaic (3DPV) structures that can generate measured energy densities (energy per base area, $\mathrm{kWh} / \mathrm{m}^{2}$ ) higher by a factor of 2-20 than stationary flat PV panels for the structures considered here, compared to an increase by a factor of 1.3-1.8 for a flat panel with dual-axis sun tracking. The increased energy density is countered by a larger solar cell area per generated energy for 3DPV compared to flat panels (by a factor of 1.5-4 in our conditions), but accompanied by a vast range of improvements. 3DPV structures can mitigate some of the variability inherent to solar $\mathrm{PV}$ as they provide a more even source of solar energy generation at all latitudes: they can double the number of peak power generation hours and dramatically reduce the seasonal, latitude and weather variations of solar energy generation compared to a flat panel design. Self-supporting 3D shapes can create new schemes for PV installation and the increased energy density can facilitate the use of cheaper thin film materials in

${ }^{a}$ Department of Materials Science and Engineering, Massachusetts Institute of Technology, 77 Massachusetts Avenue, Cambridge, MA 02139-4307, USA.E-mail: jcg@mit.edu

${ }^{b}$ Department of Mathematics, Massachusetts Institute of Technology, 77 Massachusetts Avenue, Cambridge, MA 02139-4307, USA

'Department of Architecture, Massachusetts Institute of Technology, 77 Massachusetts Avenue, Cambridge, MA 02139-4307, USA

$\uparrow$ Electronic supplementary information (ESI) available. See DOI: $10.1039 / \mathrm{c} 2 \mathrm{ee} 21170 \mathrm{j}$ area-limited applications. Our findings suggest that harnessing solar energy in three dimensions can open new avenues towards Terawattscale generation.

Converting the abundant flow of solar power to the Earth (87 PW) into affordable electricity is an enormous challenge, limited only by human ingenuity. ${ }^{1-6}$ Photovoltaic (PV) conversion has emerged as a rapidly expanding technology capable of reaching $\mathrm{GW}$-scale electric power generation with the highest power density among renewable sources of $20-40 \mathrm{~W} \mathrm{~m}^{-2} .7,8$ The main barriers to widespread adoption of PV technology include system costs (currently 3-5\$/Watt-peak) of which $\sim 60 \%$ is due to installation costs, ${ }^{9}$ the limited number of peak insolation hours available in most locations (further reduced by weather), and the requirement of a minimum threshold power density for cheaper thin-film technologies to become feasible for residential or commercial rooftop installations.

The main approach applied so far to alleviate these problems has been to search for lower-cost active layers with higher power conversion efficiencies. However, efficiency improvements can only partially reduce the installation costs and cannot change the pattern of solar energy generation, since these aspects are related to the PV system design.

A commonly adopted design consists of flat panels arranged on a flat surface - often a rooftop imposing further geometrical constraints - that yields far-from-optimal coupling with the Sun's trajectory. Sun-tracking systems can extend the range of useful peak hours, but add significant costs and are not well suited for residential or commercial installations due to the use of expensive and bulky movable parts.

\section{Broader context}

Flat photovoltaic (PV) panels deployed in rooftop installations are oriented using simple rules of thumb to optimize solar energy collection, while in large-scale solar energy generation plants sunlight collection is optimized using bulky and expensive sun trackers. However, solar energy collection largely occurs on flat structures in contrast with the strategies adopted in Nature. Here, we study the problem of how to best arrange solar panels in three dimensions to make macroscopically three-dimensional PV (3DPV) devices capable of optimizing the energy generated in a given base area (energy density). We show that 3DPV can display a range of advantages such as doubling of the peak power generation hours, and for the structures considered here a measured increase in the energy density by a factor of 2-20 in the absence of sun tracking. 3DPV also shows greatly reduced seasonal and latitude variability in the energy generation compared to flat panel design, and holds promise to reduce installation costs. Although the area of PV material per unit of generated energy compared to flat panels is higher in the case of 3DPV, given the fact that the module is no longer the main cost in PV installations and will continue to decrease relative to other costs, our results suggest that the time is ripe to fully explore the advantages of macroscopic $3 \mathrm{DPV}$. 
The flat design of PV systems contrasts with the three-dimensionality of sunlight collecting structures found in Nature. ${ }^{3,4}$ Two main physical reasons underlying the advantages of collecting light in $3 \mathrm{D}$ are the presence of multiple orientations of the absorbers that allow for the effective capture of off-peak sunlight, and the re-absorption of light reflected within the 3D structure.

We recently employed computer simulations (ref. 5) to show that 3D photovoltaic (3DPV) structures can increase the generated energy density (energy per footprint area, $\mathrm{kWh} / \mathrm{m}^{2}$ ) by a factor linear in the structure height, for a given day and location. Optimal shapes derived using a genetic algorithm approach include a cubic box open at the top and a cubic box with funnel-like shaped faces, both capable in principle of doubling the daily energy density. ${ }^{5}$ The higher area of PV material per unit of generated energy compared to flat panel designs is a main disadvantage of $3 \mathrm{DPV}$, although this is alleviated by the fact that the module is not the main cost in PV installations at present, and the PV outlay will become increasingly dominated by nonmodule costs in the near future. ${ }^{9}$ Additional practical challenges include inexpensive 3D fabrication routes and optimization of the electronics to avoid power losses.

Despite the enormous potential of macroscopic 3DPV structures, the lack of a comprehensive optimization approach and systematic study of the benefits in different seasons, locations and weather conditions, combined with the fact that the module has until only recently dominated the total cost of $\mathrm{PV}$, have thus far limited the advancement of 3DPV as a groundbreaking concept and technology.

Here, we demonstrate that 3DPV structures can be realized practically and can dramatically improve solar energy generation:10 compared to a flat panel, they can nearly double the number of peak hours available for solar energy generation, provide a measured increase in the energy density by a factor of $\sim 2-20$ without sun tracking with even higher figures in the case of cloudy weather, and reduce the large variability in solar energy generation with latitude and season found in non-tracking flat panels. 3DPV structures additionally enable the design of effective sunlight concentrators using fixed mirrors.

We establish and implement numerically a general formalism to calculate the energy generated over a period of time, at any location on Earth, by a 3D assembly of $N$ solar cells of arbitrary shape, orientation, conversion efficiency and optical properties (ESI $\dagger$ ). The calculations account for inter-cell shading, Air-Mass effects in the incident solar energy and angle-dependent reflection of unpolarized light. ${ }^{11}$ The Sun's trajectory is computed for the particular day and location using an algorithm developed by Reda et al. ${ }^{12,13}$ Weather is not explicitly taken into account in the simulations and unless otherwise stated all the simulated energy values in this work assume clear weather.

Once the 3DPV structure has been defined (for convenience broken down into triangles in our simulations), the generated energy can be expressed as an objective function of the cell coordinates and can thus be maximized using standard Monte Carlo (MC) simulated annealing and genetic algorithm (GA) optimization techniques, ${ }^{14-18}$ both implemented here. The two main forces operating during the maximization of energy generation in $3 \mathrm{D}$ are the avoidance of intercell shading and the optimization of the re-absorption of light reflected by other cells, with an intricate trade-off (dependent on the Sun's trajectory) typical of complex systems.

While here the focus is on electricity generation, the general computational approach we have implemented could allow for the optimization of a wide range of human activities that rely on sunlight collection, including heating, food crops, wine-making, and sustainable buildings.

In order to study 3DPV systems experimentally, we fabricated and tested simple 3DPV structures consisting of a cube open at the top covered by solar cells both on the interior and exterior surfaces (here referred to as an open cube structure), a similar open parallelepiped of the same base area but twice as high, and a tower with ridged faces (Fig. 1a, and ESI $\dagger$ ). The structures are made of, respectively, 9, 17 and 32 commercially available $\mathrm{Si}$ solar panels.
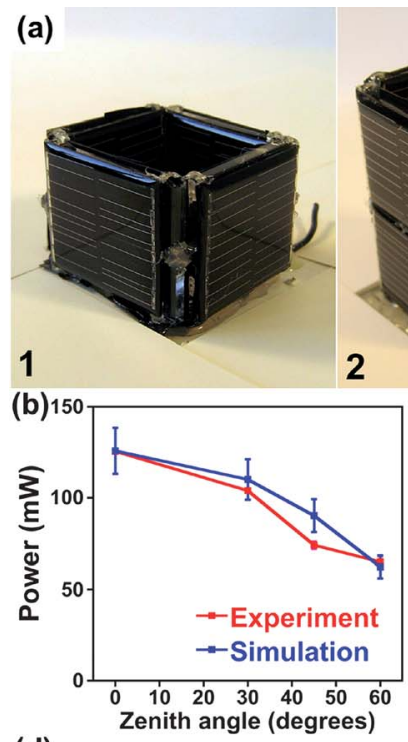

(d)
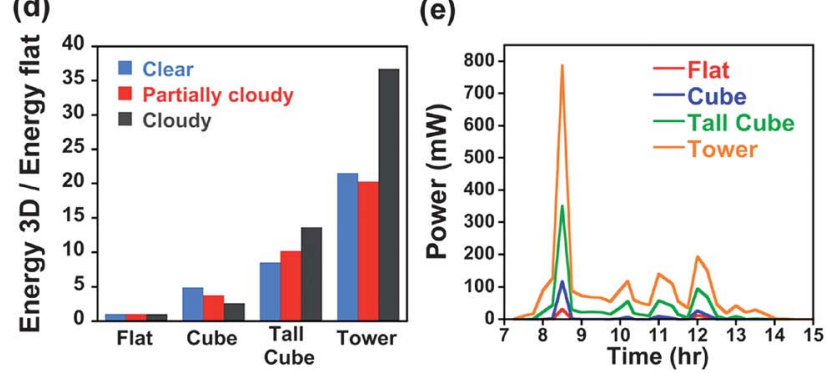

Fig. 1 (a) 3DPV structures made using Si solar cells with area $3 \times 3 \mathrm{~cm}^{2}$. From left to right, an open cube (1), an open parallelepiped twice as tall (2), and a tower (3). The structures are made up, respectively, of 9, 17, and 32 solar cells. (b) Power generated by a flat Si panel at various tilt angles measured under simulated solar light illumination, and comparison with computer simulation. The error bars in the simulation results derive from a range in the assumed efficiency of $+/-1 \%$. (c) Both measured and simulated power during a single sunny day for the open cube and for a flat panel of the same base area, showing a maximal range of hours of constant power generation and nearly twice the energy density output for the 3DPV case compared to the flat panel. (d) Energy generated by the structures shown in (a) under different weather conditions, expressed as a ratio to the energy generated by a flat panel under the same weather conditions. Comparison of the black and blue bars for the case of the parallelepiped and tower shows how structures of higher aspect ratio than the open cube can further outperform a flat panel on a cloudy day compared to a clear day. The parallelepiped in (a) is referred here as "tall cube". (e) Power generated vs. time for the data of cloudy weather shown in (d). 
Next, we measured the performance of the 3DPV structures. A flat panel was tested indoors under simulated solar light for validation of our simulations at different tilt angles to the light source (Fig. 1b), while measurements for all 3DPV shapes in Fig. 1a were collected outdoors under direct sunlight illumination (Fig. 1c-e). We validated the calculations from our computer code by comparing with experimental results for identical conditions (Fig. 1b,c and ESI $\dagger$ ) and found excellent agreement between the two, thus confirming the reliability of our code. ${ }^{19}$

The measured performance of a design as simple as the open cube under direct sunlight illumination on a summer day (Jun 16th) shows clearly the benefits of $3 \mathrm{DPV}$ compared to the conventional flat design (Fig. 1c): a daily energy generation of $2.25 \mathrm{Wh}(2.27 \mathrm{Wh}$ in the simulation) was measured for the open cube compared to $1.22 \mathrm{Wh}$ (1.01 Wh in the simulation) for a flat solar cell of the same base area under the same conditions. Such a near doubling in the energy generation resulted from an increase in both the number of hours of peak power generation and the power output throughout the day. The number of hours over which power generation was approximately constant is more than doubled for the 3DPV case compared to the flat panel, and extends between $1 \mathrm{~h}$ after sunrise and until $1 \mathrm{~h}$ before sunset.

Larger gains over a flat panel can be achieved using taller and more complex structures such as the open parallelepiped and ridged tower (Fig. 1d), with increases during the winter season even further enhanced compared to the summer. For example, the daily energy generation measured in clear weather (Fig. 1d) for a winter day (Nov. 18th) expressed as a ratio to the energy generated by a flat panel of the same base area tested under the same conditions was 4.88 for the open cube, 8.49 for the parallelepiped and 21.5 for the tower. The excess solar cell area per unit generated energy used for the 3DPV structures compared to the flat panel case was in the range of 1.5-4 for the cases examined here, with a minimum value of 1.5 corresponding to the tower case in the winter and a maximum value of $\sim 4$ for the cube in the summer.

Taller and more complex structures show an increasingly inhomogeneous cell illumination pattern with a higher number of partially shaded cells (Supplementary Movie, ESI $\dagger$ ), an effect that can introduce power $\operatorname{losses}^{20}$ and ultimately reduce the overall energy gain. We found that such power losses are mainly determined by the presence of parasitic dark currents in the shaded cells, and we were able to successfully minimize these losses with the addition of blocking diodes in series with each panel in the structure (ESI $\dagger$ ).

We used the same outdoor testing apparatus to measure the performance of 3DPV systems under different weather conditions during the same week as the clear weather results in Fig. 1d. Our data shows that the diffuse light induced by clouds, rain and mist can be captured much more efficiently in 3DPV systems compared to flat panels, leading to increased energy generation enhancement factors for cloudy weather compared to clear weather (Fig. 1d,e). The relative decrease in generated energy due to clouds is thus less significant for a 3D structure than for a flat panel and hence 3DPV systems are a source of renewable electricity less impacted by weather conditions.

In order to assess the effects of season and latitude on 3DPV performance, we studied the annual energy generation of 3DPV systems - a quantity strongly dependent on the coupling to the Sun's trajectory throughout the year - at different locations on Earth. We performed computer simulations of the energy generated by 3DPV structures over a full year at latitudes between $35^{\circ}$ South to $65^{\circ}$ North (almost all inhabited land), with an approximate latitude increase of $10^{\circ}$ between locations and for over 20 cities in the world (ESI $\dagger$ ). These results are compared with data for fixed horizontal panels (from our simulations) and for both fixed flat panels with optimal orientation and using dual-axis sun tracking (from the literature, see ref. 6).

Optimal static panel orientation can afford an increase in annual generated energy density $\left(\mathrm{kWh} / \mathrm{m}^{2}\right.$ year) compared to a flat horizontal panel by a factor of $1.1-1.25 .{ }^{6}$ Dual-axis tracking provides at present the best way to dynamically couple a PV panel to the Sun's trajectory, and can yield an increase of annual generated energy by a factor of 1.35-1.8 compared to a flat horizontal panel, ${ }^{6}$ at the cost of using expensive movable parts to track the Sun's position.

For comparison, we calculated the same ratio (defined as $Y$ here) of annual generated energy density for simple 3DPV structures to that of a flat horizontal panel of same base area, at several different latitudes (Fig. 2a). Even with a simple open cube structure, a large increase in the annual energy generation compared to a flat horizontal panel is found for 3DPV, with values of $Y$ in the range 2.1-3.8, increasing monotonically from the equator to the poles. This trend compensates the lower ground insolation at larger latitudes to give an overall density of generated energy with significantly lower variation between locations at different latitudes for the 3DPV case compared to a flat panel (Table S2 in ESI $\dagger$ ).

When compared to flat panels with optimal orientation (ref. 6, or from our calculations with similar results), an increase in the generated energy density in the range of 1.8-3 is found, thus still superior to the dual-axis tracking case.

For latitudes with maximal population density (between $50^{\circ} \mathrm{N}$ and $25^{\circ} \mathrm{N}^{21}$ values of $Y$ are in the range of 2.5-3, suggesting that $3 \mathrm{DPV}$ structures can be used to increase the energy density (and consequently enable cheaper PV technologies) in geographical areas where future PV installations will abound.

The ratio of generated energy from a 3D structure to that of a flat panel increases from summer to winter (Fig. 2b) by a larger factor at higher latitudes, implying that 3DPV has lower variation in the energy generation due to season, for the same physical reason leading to reduced latitude variability - namely, a greater ability to collect sunlight when the sun is at low elevation angles compared to a flat panel.

Further possibilities to exploit solar energy generation in 3D include incorporating mirrors together with PV panels within the structure, with the aim of concentrating sunlight without suntracking systems, in contrast to existing concentrating technologies. Structures made of a combination of mirrors and solar panels were optimized using a simulated annealing optimization scheme. The concentration ratio (a figure of merit) is defined here as a ratio between the energy per unit area of active material generated with and without mirrors. ${ }^{22}$

A highest concentration of $\sim 3.5$ was obtained for maximal mirror area within a fixed simulation volume (Fig. 3a). The best concentrating structure consisted of a solar cell cutting the body diagonal of the simulation box and enclosed within two regions of mirrors in an "open flower" configuration facing the Sun (Fig. 3b). In this highconcentration limit, the use of a given amount of PV material is optimal for the 3DPV case: the energy per unit of PV active material is almost as high as for the flat panel case, yet with an energy generation $25 \%$ higher than the latter. On the other hand, a higher mirror area causes a decrease in the generated energy density, thus 
(a)

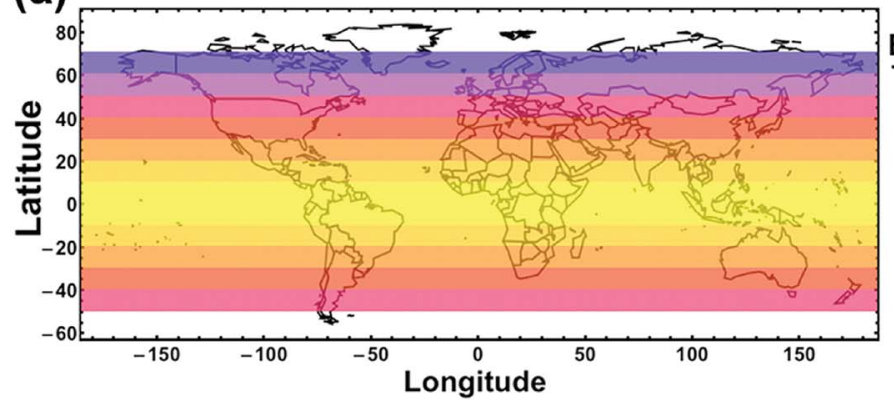

(b)

Energy cube Energy flat

3.8
3.4
2.8
2.6
2.3
2.2
2.1

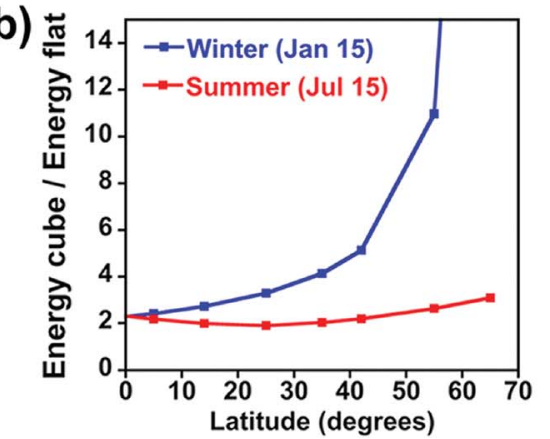

Fig. 2 (a) Density plot of the variable $Y$, defined as the ratio of the annual energy density for an open cube 3DPV structure to that of a flat horizontal panel of same base area. Values of $Y$ in the range 2.1-3.8 found here for static 3DPV structures largely exceed those predicted for dual-axis tracking. (b) Ratio of energy generated by an open cube compared to a flat panel for different seasons. 3DPV outperforms a flat panel by a larger amount during the winter and at higher latitudes due to the increased ability to use sunlight from lower elevation angles in the sky. The winter and summer labels refer to the Northern hemisphere; the curves would look the same for the Southern hemisphere provided the difference in season is taken into account.

(a)

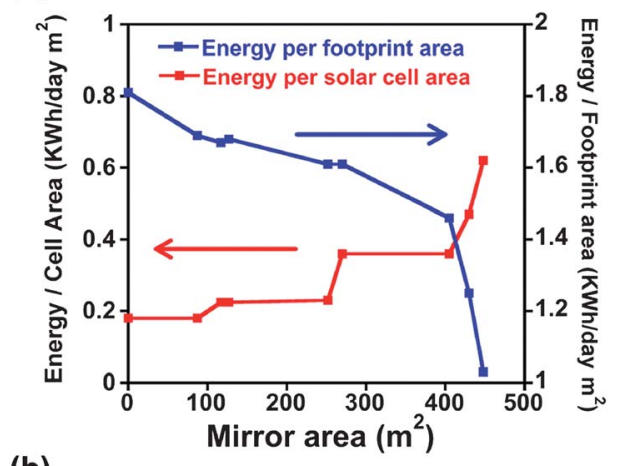

(b)
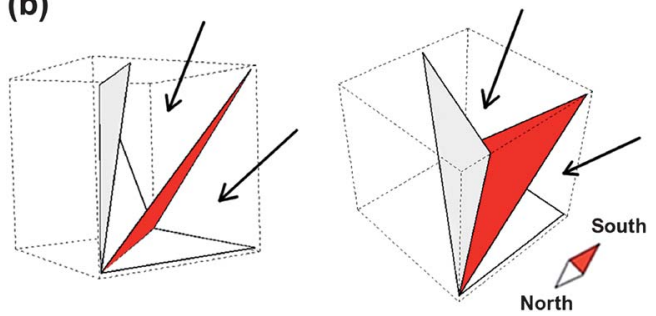

Fig. 3 (a) Concentration of light by means of mirrors is quantified by the increase in the energy per unit solar cell area. For 3D solutions provided by the MC algorithm with a $10 \mathrm{~m}$ side cubic simulation box, the red curve describes the energy obtained in a day per unit area of solar cells. In the absence of mirrors, 3DPV optimizes the energy/footprint area (blue curve) rather than the energy per solar cell area. The latter can be optimized by sunlight concentration, as seen from the opposite trend of the two curves. A maximal concentration ratio of $\sim 3.5$ is inferred by comparing the values at the two ends of the red curve. (b) Best-concentrating configuration of mirrors (light gray) and solar cells (red) in a $10 \mathrm{~m}$ side cubic volume; a simplified structure extracted from the MC optimization is shown here. It consists of a solar panel arranged between two mirrors and resembles a flower open towards the sunlight direction (South in the figure). The black arrows show the direction of incident sunlight.

defining two opposite limits for volumetric solar energy generation (Fig. 3a): maximal energy per footprint area (3DPV case) and maximal energy per active material area (flat panel case). This further elucidates the difference between sunlight collection in two and three dimensions, and illustrates the extra design flexibility inherent to the use of $3 \mathrm{D}$ structures.

In addition to intriguing fundamental aspects, 3D solar collecting structures show tremendous promise for practical applications. Potential 3DPV technologies could include structures that ship flat and expand to fill a volume in an origami-like manner, for ground or flatroof installation, or chargers for electric-powered vehicles in urban areas, or in sustainable buildings using novel semitransparent flexible PV cells incorporated in walls and windows. Two such cases are examined in detail in the ESI: $\uparrow$ a 3D electric bike charger prototype and a $50 \mathrm{~m}$ tall building with the surface completely coated with solar panels.

In closing, we observe that a comparative cost analysis between 3DPV and flat panel design is far from simple: apart from the higher number of panels used per unit energy in 3DPV, estimates of the installation costs and solar cell wiring costs are necessary, together with an estimate of the benefits of having a larger number of peak hours during the day. A detailed study would benefit from using the concept of levelized cost of energy, ${ }^{23}$ although this is beyond the scope of the present work.

In summary, the striking range of improvements imparted by three-dimensionality to static solar collecting structures stems from their optimal coupling with the Sun's trajectory. 3DPV structures using simple shapes and electrical connections largely outperform flat panels of the same base area, and show promise for embedding PV systems in the urban environment beyond the flat panel form on rooftops. Computer design facilitates the prediction of generated energy and optimal shapes, and will be an indispensable tool for optimizing solar energy generation. Our results show that $3 \mathrm{D}$ sunlight collection has the potential to serve as a paradigm shift in solar energy conversion toward the Terawatt scale.

\section{Acknowledgements}

The authors are grateful to Vladimir Bulovic for many useful discussions. Some of the calculations were carried out at Teragrid computing facilities.

\section{References}

1 D. Ginley, M. A. Green and R. Collins, MRS Bull., 2008, 33, 355.

2 M. Mehos, MRS Bull., 2008, 33, 364. 
3 W. W. Robbins, T. E. Weier and C. R. Stocking, Botany: an Introduction to Plant Science 3rd Ed., Wiley, New York, 1965.

4 R. E. Blankenship, et al., Science, 2011, 332, 805.

5 B. Myers, M. Bernardi and J. C. Grossman, Appl. Phys. Lett., 2010, 96, 071902. It is important to distinguish between the use of macroscopic three-dimensionality in solar cells, the topic of this reference as well as the present manuscript, and nanoscale three dimensional structures to enhance light trapping at the surface of flat panel solar cells.

6 M. Lave and J. Kleissl, Renewable Energy, 2011, 36, 1145. A similar database for all latitudes is not available to our knowledge.

7 REN 21, Renewables 2010 Global Status Report, 19 (2010). See www.ren21.net.

8 V. Smil, Energy: a Beginner's Guide, Oneworld, Glasgow, Great Britain, 2006.

9 G. Barbose, N. Darghouth and R. Wiser, Report LBNL-4121E (2010). See http://eetd.lbl.gov/ea/emp/re-pubs.html.

10 D. Perreault, V. Bulovic, J. C. Grossman, M. Bernardi and N. Ferralis, Three-Dimensional Photovoltaic Apparatus and MethodPatent No. WO 2011/097485.

11 J. D. Jackson, Classical Electrodynamics 3rd Ed., Wiley, New York, USA, 1998.

12 I. Reda and A. Andreas, Sol. Energy, 2004, 76, 577.

13 See also http://www.pveducation.org/pvcdrom/properties-ofsunlight/sun-position-calculator.

14 R. W. Shonkwiler and F. Mendivil, Explorations in Monte Carlo Methods, Springer, New York, USA, 2009.

15 M. Mitchell, An Introduction to Genetic Algorithms, MIT Press, Boston, USA, 1998.
16 S. Kumara, Single and Multiobjective Genetic Algorithm Toolbox in C++.

17 D. T. Pham and D. Karaboga, Intelligent Optimization Techniques, Springer-Verlag, London, England, 2000.

18 H. Hernandez-Noyola, D. H. Potterveld, R. J. Holt and S. B. Darling, Energy Environ. Sci., 2012, 5, 5798-5802.

19 The deviation between the experimental and the calculated curve seen during the early morning and late afternoon hours (Fig. 1c) is likely due to the collection of diffuse light in the experiment. At the solar noon, the simulated power shows a marked dip for the open cube case, also present to a smaller extent in the experiment. Such difference can be attributed to the ideality of the simulated structure, where the infinitesimal cell thickness and the ideal dueSouth orientation completely cancel the contribution from side cells in the simulation at the solar noon, and also to electrical effects. For these reasons, the very small discrepancy $(1 \%)$ found for the energy generated by the cube is a result of error compensation, while a discrepancy between the simulation and the experiment of the order of $5-15 \%$ is not uncommon even when the main physical effects have been captured in the simulation, as in the flat panel case in Fig. 1c.

20 A. Woyte, J. Nijs and R. Belmans, Sol. Energy, 2003, 74, 217.

21 W. Tobler, Proceedings, Auto Carto 13, Seattle (April 1997).

22 It must be noted that 3DPV does not normally optimize the energy per unit area of active material, but rather optimizes the energy generated from a given volume and hence for a given footprint area.

23 S. B. Darling, F. You, T. Veselka and A. Velosa, Energy Environ. Sci., 2011, 4, 3133-3139. 